\title{
Symbolic Dynamic Analysis of Relations Between Cardiac and Breathing Cycles in Patients on Weaning Trials
}

\author{
P. Caminal, ${ }^{1,2,3}$ B. F. Giraldo, ${ }^{1,3,4}$ M. Vallverdú, ${ }^{1,2,3}$ S. Benito, ${ }^{5}$ R. Schroeder, ${ }^{6}$ and A. Voss ${ }^{6}$ \\ ${ }^{1}$ Departament ESAII, Universitat Politècnica de Catalunya (UPC), Pau Gargallo, 5, 08028 Barcelona, Spain; ${ }^{2}$ Biomedical \\ Engineering Research Centre (CREB), Universitat Politècnica de Catalunya (UPC), Barcelona, Spain; ${ }^{3}$ CIBER de Bioingeniería, \\ Biomateriales y Nanomedicina (CIBER-BBN), Barcelona, Spain; ${ }^{4}$ Institut de Bioenginyeria de Catalunya (IBEC), Barcelona, \\ Spain; ${ }^{5}$ Hospital de la Santa Creu i Sant Pau, Barcelona, Spain; and ${ }^{6}$ Department of Medical Engineering and Biotechnology, \\ University of Applied Sciences Jena, Jena, Germany
}

(Received 30 July 2009; accepted 25 March 2010; published online 20 April 2010)

Associate Editor Kenneth R. Lutchen oversaw the review of this article.

\begin{abstract}
Traditional time-domain techniques of data analysis are often not sufficient to characterize the complex dynamics of the cardiorespiratory interdependencies during the weaning trials. In this paper, the interactions between the heart rate (HR) and the breathing rate (BR) were studied using joint symbolic dynamic analysis. A total of 133 patients on weaning trials from mechanical ventilation were analyzed: 94 patients with successful weaning (group S) and 39 patients that failed to maintain spontaneous breathing (group F). The word distribution matrix enabled a coarse-grained quantitative assessment of short-term nonlinear analysis of the cardiorespiratory interactions. The histogram of the occurrence probability of the cardiorespiratory words presented a higher homogeneity in group $\mathrm{F}$ than in group $\mathrm{S}$, measured with a higher number of forbidden words in group $\mathrm{S}$ as well as a higher number of words whose probability of occurrence is higher than a probability threshold in group $\mathrm{S}$. The discriminant analysis revealed the best results when applying symbolic dynamic variables. Therefore, we hypothesize that joint symbolic dynamic analysis provides enhanced information about different interactions between HR and BR, when comparing patients with successful weaning and patients that failed to maintain spontaneous breathing in the weaning procedure.
\end{abstract}

Keywords-Dynamical nonlinearities analysis, Cardiorespiratory interdependencies, Joint symbolic dynamic, Weaning procedure.

\section{INTRODUCTION}

Acute respiratory, cardiac, and neurologic failures lead to intubation and mechanical ventilation support. Withdrawal of mechanical ventilation should be

Address correspondence to B. F. Giraldo, Departament ESAII, Universitat Politècnica de Catalunya (UPC), Pau Gargallo, 5, 08028 Barcelona, Spain. Electronic mail: Beatriz.Giraldo@upc.edu performed as soon as autonomous respiration can be sustained. This process is usually done by the gradual removal of the mechanical support as spontaneous breathing is resumed, ${ }^{2}$ and is referred to as weaning. Both an unnecessary delay in the discontinuation process $^{39}$ and a weaning trial undertaken too early ${ }^{18}$ are undesirable. When mechanical ventilation is discontinued, up to $25 \%$ of patients have respiratory distress severe enough to require reinstitution of ventilatory support. ${ }^{34}$ Different studies have been performed in order to detect which physiological variables identify readiness to undertake a weaning trial. Most of the information collected in these studies are synthesized in several articles, ${ }^{8,11,13,28,36}$ which may establish guidelines for weaning criteria and protocols. It was also hypothesized that the variability of the respiratory pattern could be a convenient weaning criterion to reduce the number of patients not successfully weaned. ${ }^{10}$ However, one of the most challenging problems in intensive care is still the process of discontinuing mechanical ventilation, because the number of patients that have to be reintubated before $48 \mathrm{~h}$ but previously performed successful trials during the 30-min test represents a percentage higher than $10 \%$. A failed weaning trial is discomforting for the patient, may induce cardiopulmonary distress and carries a higher mortality rate. ${ }^{7,30}$ Many controversial questions remain concerning the best methods for conducting this process. ${ }^{7}$ Further study is needed of the effectiveness in the weaning process. ${ }^{4}$

Assessing autonomic control provides information about patho-physiological imbalances within the cardiorespiratory system. The cardiac and the respiratory system are controlled by two interacting self-sustained oscillators, each having its own period. It is convenient 
to analyze the cardiorespiratory interdependencies from the time series of the cardiac interbeat intervals and the time series of the breath duration, which correspond to the periods of both oscillators. The different cardiorespiratory interdependencies during the weaning trials are particular aspects of dynamic autonomic functional coordination. Up to now, it is not clear whether there are more stable functional relations between breaths and heart beats in patients with successful trials. As the coupling between heart rate (HR) and respiration is assumed to be strongly nonlinear, several methods have been developed to analyze the cardiorespiratory coordination. Assuming that the HR and the respiratory frequency are two self-sustained oscillators, measures of phase synchronization and its coupling strength can be derived. ${ }^{21,29,35}$ As an enhancement of crosscorrelation, mutual information has been introduced to analyze the linear and nonlinear dynamics of the cardiorespiratory system. ${ }^{16,23}$ Information theory has also been used to analyze causal relationships between the cardiac and respiratory oscillations dur-

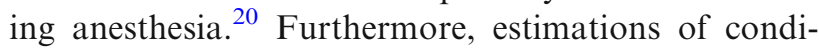
tional entropy, predictability, and correlation dimension have been applied to assess the coupling strength. ${ }^{14,15}$ The synchronization analysis has been utilized to measure the coupling strength between the beat-to-beat variability of the sympathetic discharge and ventilation in decerebrate artificially ventilated cats. ${ }^{24,26}$ Experimental data from various studies on autonomic coordination, in healthy humans, patients after myocardial infarction and patients with sleep disorders, have emphasized the importance of multimodal assessment. ${ }^{1,9,12,22}$

In this paper, we introduce the joint symbolic dynamic analysis of heart rate (HR) and breathing rate (BR) that may improve the understanding of the physiological processes involved in the weaning procedure. In this way, the study of dynamics simplifies to the description of bivariate symbol sequences. Some detailed information has been lost, but some of the invariant, robust properties of the dynamics have been kept. Univariate symbolic dynamics has been already successfully applied in HR variability analysis ${ }^{37,38}$ and respiratory pattern analysis. ${ }^{6}$ Bivariate symbolic dynamic analysis has provided an efficient nonlinear representation of $\mathrm{HR}$ and systolic blood pressure interactions that offers simple physiological interpretations. ${ }^{3}$ In order to apply joint symbolic dynamics, the values of the cardiac interbeat durations and the breath durations had to be synchronized. Since electrocardiographic and respiratory flow signals can be measured noninvasively, this analysis may be advantageous in future automatic diagnostic of patients during the weaning trials.
Therefore, we hypothesize that joint symbolic dynamic analysis provides enhanced information about different interactions between HR and BR, when comparing patients with successful trials and patients that failed to maintain spontaneous breathing in the weaning procedure. A preliminary version of this work was reported by Caminal et al. ${ }^{5}$

\section{METHODS}

\section{Patients and Data Preprocessing}

Electrocardiographic (ECG) and respiratory flow signals were measured in 133 patients on weaning trials from mechanical ventilation (WEANDB data base). These patients were recorded in the Departments of Intensive Care Medicine at Santa Creu i Sant Pau Hospital and Getafe Hospital, according to the protocols approved by the local ethic committees and with informed consent obtained.

Using clinical criteria based on the T-tube test, the patients were included in this study according with the standard indices (clinical criteria) that decide to begin the spontaneous breathing test: resolution of the etiology of respiratory failure (with inspired oxygen fraction $\left[\mathrm{FIO}_{2}\right] \leq 0.4$, oxygen saturation $\left[\mathrm{SO}_{2}\right] \geq 90 \%$ and need for positive end-expiratory pressure [PEEP $\leq 5 \mathrm{~cm}$ to $\left.\mathrm{H}_{2} \mathrm{O}\right]$ ), hemodynamic stability (absence of myocardium ischemia and/or heart failure, cardiac frequency $\leq 140 \mathrm{bpm}$, and stable arterial tension with least tolerating a reduction in inotropic support), and adequate respiratory muscle function (acceptable respiratory rate). All patients had a stable sinus rhythm during recording, with a number of ectopic beats of 0.05 [0.01 0.24$] \%$ (median [interquartile range]).

The patients were submitted under a test of spontaneous breathing, were disconnected from the ventilator, and maintained spontaneous breathing through an endotraqueal tube during $30 \mathrm{~min}$. If the patients maintained the spontaneous breathing with normality they were extubated, if not, they were reconnected. When the patients still maintained the spontaneous breathing after $48 \mathrm{~h}$, the weaning trial process was considered successful, if not, the patients were reintubated (Fig. 1).

In this study, we investigated two patient groups: Group S, 94 patients (61 male, 33 female) with successful weanings (maintained spontaneous breathing after $48 \mathrm{~h}$ ) and Group F, 39 patients (24 male, 15 female) who failed to maintain spontaneous breathing and were reconnected after $30 \mathrm{~min}$ of weaning trials. The patient groups were of comparable age (Group S: $65 \pm 17$ years; 
Group F: $67 \pm 15$ years). This study, however, does not include reintubated patients because of the low number of subjects that does not allow statistical significance analysis.

The ECG signal was recorded using a SpaceLabs Medical monitor. Respiratory flow signal was obtained using a pneumotachograph connected to an endotraqueal tube. The pneumotachograph consists on a Datex-Ohmeda monitor with a Variable-Reluctance Transducer (Validyne Model MP45-1-871, Validyne Corp., Northridge, CA). Both signals were recorded at a sampling frequency of $250 \mathrm{~Hz}$ during $30 \mathrm{~min}$. Time series of the cardiac interbeat intervals $R R\left(k_{1}\right)$ were extracted automatically from the ECG signal using an algorithm based on wavelet analysis. ${ }^{19}$ Ectopic beats were determined, removed, and interpolated using an algorithm based on local variance estimation. Time series of the breath durations $T_{T o t}\left(k_{2}\right)$ were extracted automatically using an algorithm based on the zerocrossing of the respiratory flow signal and, thereafter, visually inspected, and if necessary, edited.

\section{Joint Symbolic Dynamics}

The concept of Symbolic Dynamics is based on the elimination of detailed information, in order to keep the robust properties of the dynamics by a coarsegraining of the measurements. ${ }^{17,38}$ In order to apply joint symbolic dynamics the values of the cardiac $R R\left(k_{1}\right)$ and respiratory $T_{T o t}\left(k_{2}\right)$ series had to be synchronized. $^{3}$ A linear interpolation method was considered. Figure $2 \mathrm{a}$ indicates the function $R R\left(k_{1}\right)$ where each one of the $R R$ values were assigned at the time where the cardiac interbeat interval was measured. Figure $2 \mathrm{~b}$ indicates the function $T_{T o t}\left(k_{2}\right)$ where each one of the $T_{T o t}$ values were assigned at the time where the breath duration was measured. New $R R(n)$ and $T_{T o t}(n)$ were calculated by synchronous sampling of the interpolation of the previous $R R\left(k_{1}\right)$ and $T_{T o t}\left(k_{2}\right)$ series (Fig. 3). Two sampling frequencies were considered: $1 \mathrm{~Hz}$ and $2 \mathrm{~Hz}$.

If $x$ is the bivariate sample vector of the cardiac $(c)$ time series $R R(n)$ and the respiratory $(r)$ time series

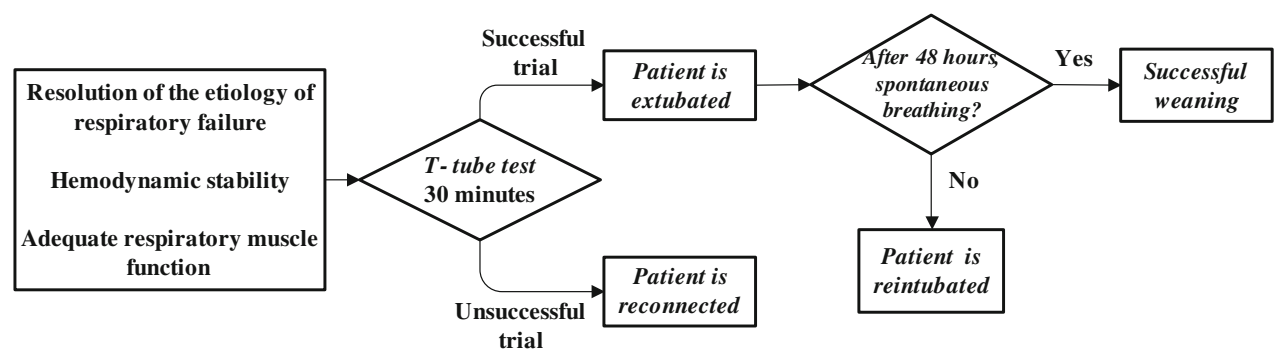

FIGURE 1. Weaning trial process.
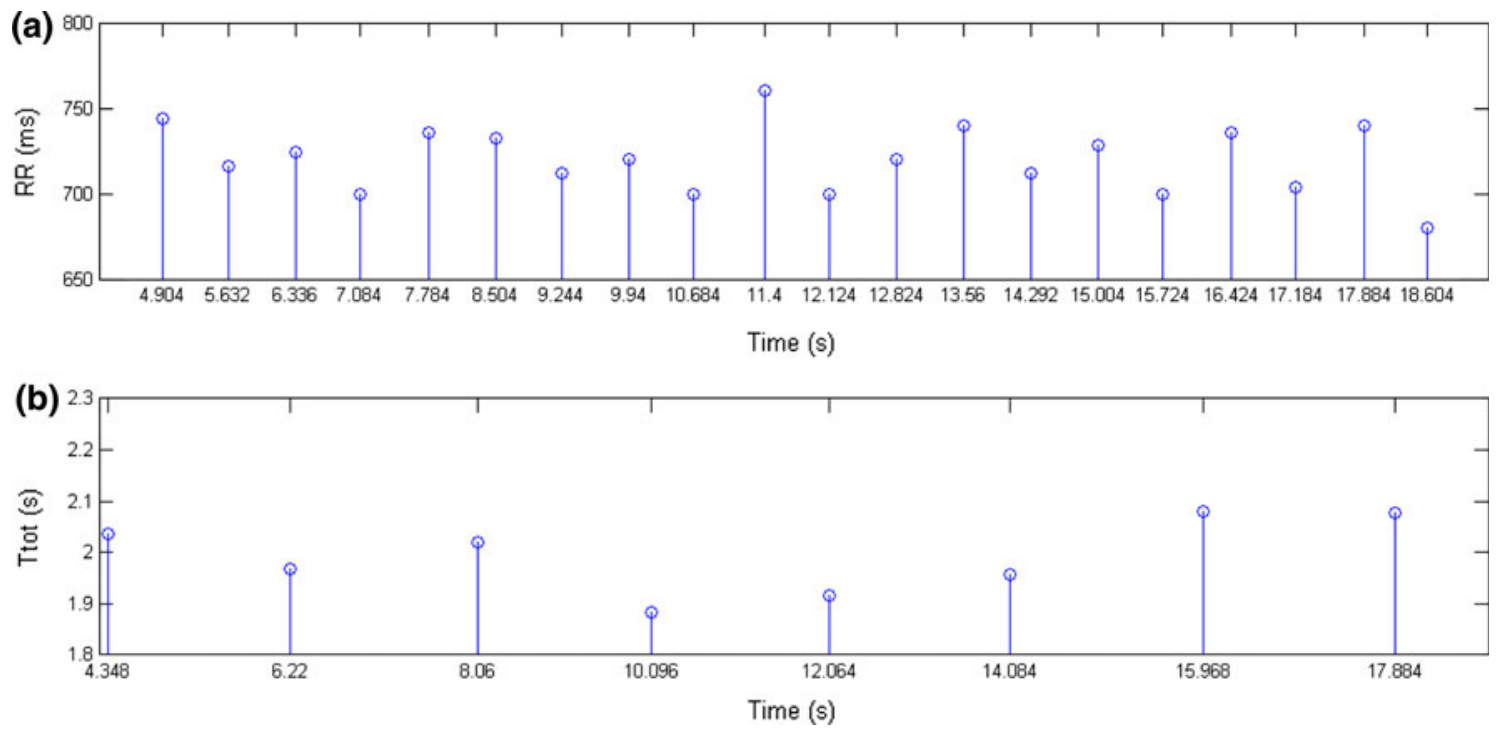

FIGURE 2. (a) Cardiac interbeat interval series $R R\left(k_{1}\right)$ vs. time and (b) breath duration series $T_{T o t}\left(k_{2}\right)$ vs. time. 

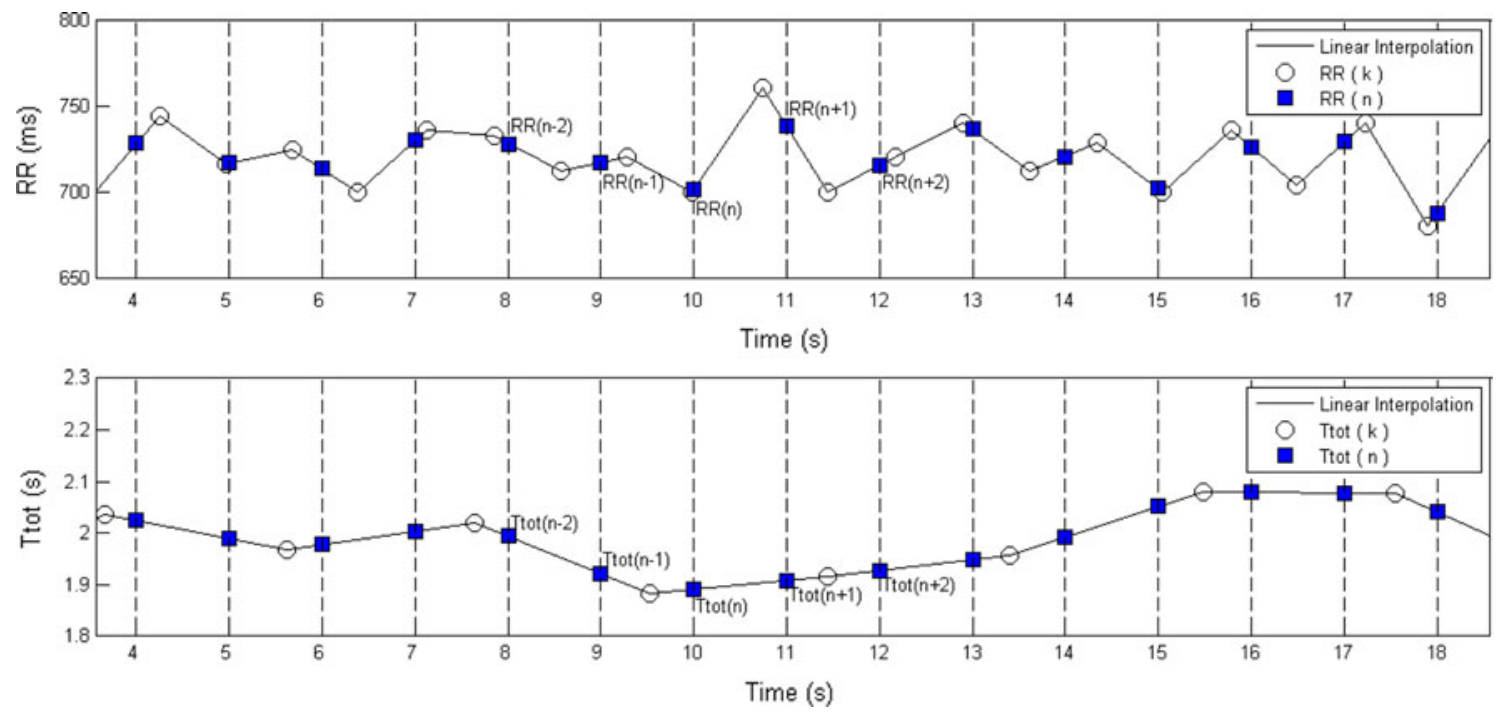

FIGURE 3. New $R R(n)$ and $T_{\text {Tot }}(n)$ time series calculated by synchronized sampling of the interpolated $R R\left(k_{1}\right)$ and $T_{T o t}\left(k_{2}\right)$ time series. Sampling frequency $1 \mathrm{~Hz}$.

$T_{T o t}(n)(1)$, then $s$ represents a bivariate symbol vector (2), gained by transforming $x$ using a symbol alphabet according to (3) and (4).

$$
\begin{gathered}
x=\left\{\left[x_{n,}^{c} x_{n}^{r}\right]^{\mathrm{T}}\right\}_{n=0,1, \ldots} \quad x \in R \\
s=\left\{\left[s_{n}^{c} s_{n}^{r}\right]^{\mathrm{T}}\right\}_{n=0,1, \ldots} \quad s \in\{0,1\} \\
s_{n}^{c}=0:\left(x_{n+1}^{c}-x_{n}^{c}\right) \leq 0 \\
1:\left(x_{n+1}^{c}-x_{n}^{c}\right)>0 \\
s_{n}^{r}=0:\left(x_{n+1}^{r}-x_{n}^{r}\right) \leq 0 \\
1:\left(x_{n+1}^{r}-x_{n}^{r}\right)>0
\end{gathered}
$$

Thus, the bivariate symbol vector $s$ consists of $a=4$ symbols. Further, $s$ was fractionalized to words (bins) $w_{k}$ of length $k .{ }^{19}$ The maximum length of the words was restricted by the probability of occurrence $p\left(w_{k}\right)$ of each word type and therefore, indirectly, by the number of measured samples. The number of overlapped symbols $\tau$ in consecutive words had to be considered for three possible values $(0,1$, and 2$)$. With $30 \mathrm{~min}$ recording time and the considered sampling frequencies, $x$ contained 1800 and 3600 samples, and thus $s$ had a length $N$ of 1798 and 3598 when considering two overlapped symbols, 900 and 1800 when considering one overlapped symbol, and 600 and 1200 when considering no overlapped symbols. As words with a length greater than three would be statistically insufficiently represented $(5),{ }^{19,38}$ the maximum length of words was limited to $k=3$, spanning over an $8 \times 8$

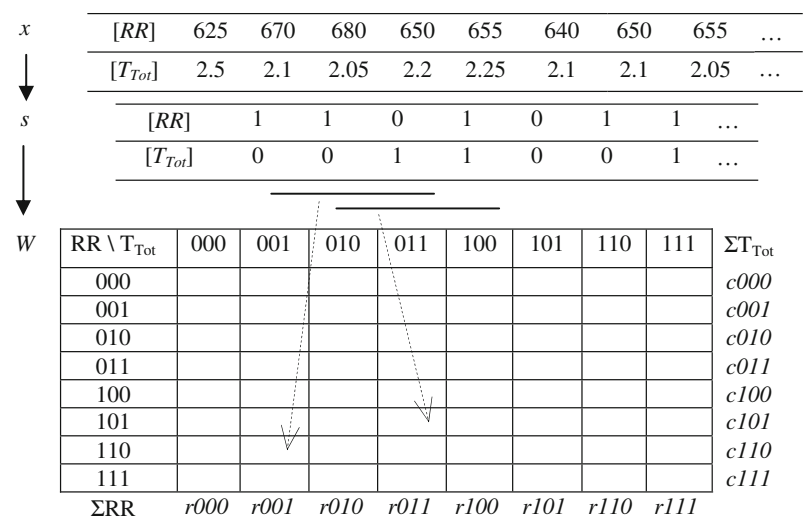

FIGURE 4. Transformation of vector $x$ containing bivariate cardiac and respiratory samples into symbol vector $s$ and word distribution matrix $W . x$ : cardiac $R R(n)$ in milliseconds/ respiratory $T_{T o t}(n)$ in seconds. $s$ : symbol 1 -increasing values/symbol $0-$ decreasing values. $W$ : row-symbol sequence of cardiac changes; column-symbol sequence of respiratory changes.

vector matrix $W$, from word type $[000,000]^{\mathrm{T}}$ to $[111,111]^{\mathrm{T}}$ (Fig. 4). From this point of view, the optimum number of overlapped symbols in consecutive words was two.

$$
p\left(w_{k}\right)=N / a^{k}
$$

To compare the word-type distributions between data sets of different length, the sum of all counted words was normalized to 1 . The normalized probabilities of all single word types' occurrences were computed as $p_{n}\left(w_{c r}\right)$. Figure 5 shows an example of three-dimensional plots of the probability distribution density matrix $W$ for a patient of group $\mathrm{S}$ and a patient of group F. 


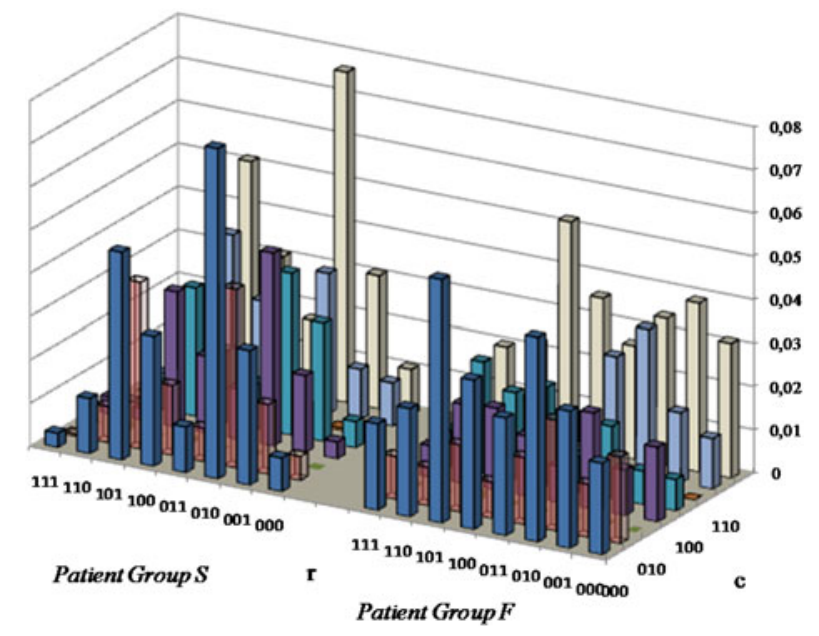

FIGURE 5. Examples of word distribution density in a patient of group $S$ and a patient of group $F . c=$ behavior of three successive $R R(n)$ changes and $r=$ behavior of three successive $T_{T o t}(n)$ changes.

The sum of each row in $W$ was computed as $p_{n}\left(w_{c}\right)$, and it corresponds to the occurrence probability of each word from the cardiac series (6). The sum of each column in $W$ was computed as $p_{n}\left(w_{r}\right)$, and it corresponds to the occurrence probability of each word from the respiratory series (7).

$$
p_{n}\left(w_{c}\right)=\sum_{r} p_{n}\left(w_{c r}\right)
$$

where $r$ takes values from " 000 " to " 111 "

$$
p_{n}\left(w_{r}\right)=\sum_{c} p_{n}\left(w_{c r}\right)
$$

where $c$ takes values from " 000 " to " 111 "

Several indexes were introduced in order to differentiate the groups of patients:

- probability of occurrence $p_{n}\left(w_{c r}\right)$ of each one of the single word types,

- probability of occurrence of each word from the cardiac series $p_{n}\left(w_{c}\right)$

- probability of occurrence of each word from the respiratory series $p_{n}\left(w_{r}\right)$,

- number of words $w_{c r} T H$ whose probability of occurrence $p_{n}\left(w_{c r}\right)$ is higher than a probability threshold $T H$,

- number of words $w_{c} T H$ whose probability of occurrence $p_{n}\left(w_{c}\right)$ is higher than a probability threshold $T H$,

- number of words $w_{r} T H$ whose probability of occurrence $p_{n}\left(w_{r}\right)$ is higher than a probability threshold $T H$,
- number of forbidden words $f w_{c r} T H$ whose probability of occurrence $p_{n}\left(w_{c r}\right)$ is lower than a probability threshold $f T H$,

- number of forbidden words $f w_{c} T H$ whose probability of occurrence $p_{n}\left(w_{c}\right)$ is lower than a probability threshold $f T H$, and

- number of forbidden words $f w_{r} T H$ whose probability of occurrence $p_{n}\left(w_{r}\right)$ is lower than a probability threshold $f T H$.

In order to define the most suitable probability thresholds, different ranges of values were considered:

- from $1 \%$ to $5 \%$ for the probability threshold $T H$ when considering $p_{n}\left(w_{c r}\right)$,

- from $5 \%$ to $25 \%$ for the probability thresholds $T H$ when considering $p_{n}\left(w_{c}\right)$ and $p_{n}\left(w_{r}\right)$,

- from $0.1 \%$ to $0.3 \%$ for the probability threshold $f T H$ when considering $p_{n}\left(w_{c r}\right)$,

- from $1 \%$ to $3 \%$ for the probability thresholds $f T H$ when considering $p_{n}\left(w_{c}\right)$ and $p_{n}\left(w_{r}\right)$.

To compare the estimated parameters between the groups of patients simple statistics were computed, including group mean, standard deviation and a Mann-Whitney two-tailed test. The (global) significance level was set as $p_{\mathrm{g}}=0.05$. Considering the problem of multiple testing, the necessary (local) significance level $\left(p_{1}\right)$ of a single parameter from an observed $v$ dimensional parameter space had to fulfill Bonferroni's inequality $^{33}$ (8) to guarantee (global) significance, as follows:

$$
p_{1}<p_{\mathrm{g}} / v
$$

In our case, for $v=64$ word types and $v=16$ row and column sums, the local significance levels were $p_{1}=0.00078$ and $p_{1}=0.0031$, respectively.

\section{Surrogate Data Approach and Deterministic Pattern Detection}

A surrogate data approach ${ }^{25,32}$ can be useful to test the significant presence of patterns. This approach allows the setting of a threshold of significance and the rejection of the null hypothesis that a pattern is present only by chance. This approach can be helpful in testing the significant presence of any word in cardiac and respiratory series. From one original cardiac series, 15 surrogate data series were obtained by randomly shuffling the samples according to 15 different white noise realizations, thus completely destroying the original power spectrum but maintaining sample distribution. The normalized probabilities of all single word types' occurrences $p_{n}\left(w_{c}\right)$ calculated over the original 
series $p_{n}\left(w_{c}\right)_{o}$ were compared with those obtained from the surrogate series $p_{n}\left(w_{c}\right)_{s}$ by using ${ }^{32}$

$$
\sigma=\left|\frac{p_{n}\left(w_{c}\right)_{o}-a v\left[p_{n}\left(w_{c}\right)_{s}\right]}{s d\left[p_{n}\left(w_{c}\right)_{s}\right]}\right|,
$$

where $a v\left[p_{n}\left(w_{c}\right)_{s}\right]$ and $s d\left[p_{n}\left(w_{c}\right)_{s}\right]$ represented the mean and the standard deviation of $p_{n}\left(w_{c}\right)_{s}$ over the 15 surrogate series. If $\sigma>2, p_{n}\left(w_{c}\right)_{o}$ could not derive from a white noise with the same sample distribution of the original series $(p<0.05)$. The same procedure was applied to one original respiratory series, comparing $p_{n}\left(w_{r}\right)_{o}$ with those obtained from the surrogate series $p_{n}\left(w_{r}\right)_{s}$.

In the case of bivariate joint symbolic analysis, the null hypothesis might be the full uncoupling between cardiac and respiratory series and surrogate series were constructed by fully decoupling the two series while maintaining their power spectra. The normalized probabilities of all single word types' occurrences $p_{n}\left(w_{c r}\right)$ calculated over the original series $p_{n}\left(w_{c r}\right)_{o}$ were also compared with those obtained from the surrogate series $p_{n}\left(w_{c r}\right)_{s}$.

\section{RESULTS}

First, time-domain analysis of the time series was performed. Table 1 shows the resulting mean values $\bar{x}$, standard deviations $S D(x)$ and interquartile ranges $\operatorname{IQR}(x)$ of the $R R\left(k_{1}\right)$ and $T_{T o t}\left(k_{2}\right)$ series obtained by comparing the patient groups $\mathrm{S}$ and $\mathrm{F}$. The mean values of the cardiac interbeat intervals $R R\left(k_{1}\right)$ and the breath duration $T_{T o t}\left(k_{2}\right)$ presented the most significant differences.

Heart rate variability indices have also been analyzed considering the frequency domain methods. ${ }^{31} \mathrm{No}$ statistical significant differences have been obtained

TABLE 1. Mean, standard deviation $S D(x)$ and interquartile range $I Q R(x)$ of the interbeat durations (RR), breath durations $\left(T_{T o t}\right)$, heart rate variability indices considering HF power and the LF/HF ratio values in normalized units ( $\mathrm{nu}$ ), and crosscorrelation coefficient for a lag of $0\left(\rho_{\mathrm{rc}}(0)\right)$.

\begin{tabular}{lccl}
\hline & $\begin{array}{c}\text { Group S, } \\
\text { mean } \pm \text { SD }\end{array}$ & $\begin{array}{c}\text { Group F, } \\
\text { mean } \pm \text { SD }\end{array}$ & $p$ Value \\
\hline Mean RR (ms) & $701 \pm 122$ & $634 \pm 101$ & 0.004 \\
SD (RR) (ms) & $72 \pm 80$ & $52 \pm 35$ & n.s. \\
IQR (RR) (ms) & $73 \pm 78$ & $60 \pm 58$ & n.s. \\
Mean $T_{\text {Tot }}(\mathrm{s})$ & $2.80 \pm 0.73$ & $2.26 \pm 0.79$ & $<0.0001$ \\
SD $\left(T_{\text {Tot }}\right)(\mathrm{s})$ & $0.85 \pm 0.96$ & $0.58 \pm 0.43$ & 0.034 \\
IQR $\left(T_{\text {Tot }}\right)(\mathrm{s})$ & $0.50 \pm 0.30$ & $0.39 \pm 0.31$ & 0.012 \\
$\mathrm{HF}($ nu) & $43 \pm 16$ & $46 \pm 16$ & n.s. \\
$\operatorname{LF} / \mathrm{HF}$ rate & $1.92 \pm 2$ & $1.55 \pm 1$ & n.s. \\
$\rho_{\text {rc }}(0)$ & $0.92 \pm 0.13$ & $0.94 \pm 0.09$ & n.s. \\
\hline
\end{tabular}

when comparing both groups of patients. Table 1 shows the resulting $\mathrm{HF}$ power and the $\mathrm{LF} / \mathrm{HF}$ ratio values in normalized units.

The linear relation between cardiac and breath cycles was analyzed using the cross-correlation coefficient $\rho_{c r}$, defined as the cross-correlation function between $R R(n)$ and $T_{T o t}(n)$ divided by the square root of the product of the auto-correlation functions of $R R(n)$ and $T_{T o t}(n)$. Different lags $(-10, \ldots, 0, \ldots 10)$ were considered. No-one of the lags presented statistical significant differences when comparing both groups of patients. Table 1 shows the mean \pm standard deviation of the $\rho_{c r}$, for a lag of $0\left(\rho_{c r},(0)\right)$.

In the symbolic dynamic analysis, the first parameter to be determined was the number of overlapped symbols in consecutive words, $\tau$, from the three possible values $(0,1$, and 2$)$. For each one of the considered sampling frequencies, the number of words whose probability of occurrence statistically differentiate between groups $\mathrm{S}$ and $\mathrm{F}$ was calculated. The optimum $\tau$ is the value that obtains the highest number of significant words, and this was $\tau=2$. A similar analysis was followed to determine the optimum sampling frequency of the $R R(n)$ and $T_{T o t}(n)$ series, considering $\tau=2$. The optimum sampling frequency to be considered in the following analysis was $f_{\mathrm{s}}=1 \mathrm{~Hz}$.

Considering $f_{\mathrm{s}}=1 \mathrm{~Hz}$, the four most dominant words were the same in both groups of patients: $c 010$ $r 111\left(p_{n}\left(w_{c 010} r_{111}\right)=0.063\right.$ and 0.054 in groups $\mathrm{S}$ and $\mathrm{F}$, respectively), c101 $r 111\left(p_{n}\left(w_{c 101} r_{111}\right)=0.056\right.$ and $0.050), c 010 r 000\left(p_{n}\left(w_{c 010} r 000\right)=0.052\right.$ and 0.044$)$, and $c 101$ r000 $\left(p_{n}\left(w_{c 101}\right.\right.$ r.000 $)=0.046$ and 0.041$)$. The probability of occurrence of these four dominant words was higher in group $\mathrm{S}$ than in group $\mathrm{F}$, showing a more deterministic structure of the cardiorespiratory interaction in the group of patients with successful trials during the weaning procedure. Furthermore, 10 of the 64 word types $(15 \%)$ obtained a cumulative probability of occurrence higher than $40 \%$ in both groups of patients. It means the presence of deterministic structures in the cardiorespiratory coupling, otherwise stochastically independent $R R(n)$ and $T_{T o t}(n)$ time series would result in a more or less equal distribution.

Table 2 shows those words whose probability of occurrence better discriminate between groups $\mathrm{S}$ and $\mathrm{F}$, when considering the sampling frequency $f_{\mathrm{s}}=1 \mathrm{~Hz}$. When comparing both groups, 12 of the 64 word types presented a global statistical significance $p_{\mathrm{g}}<0.05$. In 11 of these significant word types the probability of occurrence was nearly three times higher in group $\mathrm{F}$ than in group $\mathrm{S}$.

Considering the row and column sum probabilities, significant differences between groups $\mathrm{S}$ and $\mathrm{F}$ occurred in all of the eight word types from the 
TABLE 2. Words whose probability of occurrence best discriminate between both patient groups $S$ and $F$ using joint symbolic dynamic analysis.

\begin{tabular}{lccc}
\hline & $\begin{array}{c}\text { Group S, } \\
\text { mean } \pm \text { SD }\end{array}$ & $\begin{array}{c}\text { Group F, } \\
\text { mean } \pm \text { SD }\end{array}$ & $p_{\mathrm{g}}$-value \\
\hline$p_{n}\left(w_{\text {c010 ro10 }}\right)$ & $0.0012 \pm 0.0021$ & $0.0035 \pm 0.0037$ & 0.0014 \\
$p_{n}\left(w_{\text {c010 r101 }}\right)$ & $0.0017 \pm 0.0028$ & $0.0045 \pm 0.0044$ & 0.0122 \\
$p_{n}\left(w_{c 110 \text { ro10 }}\right)$ & $0.0005 \pm 0.0014$ & $0.0019 \pm 0.0029$ & 0.0103 \\
$p_{n}\left(w_{\text {c001 r010 }}\right)$ & $0.0005 \pm 0.0011$ & $0.0019 \pm 0.0022$ & 0.0007 \\
$p_{n}\left(w_{c 100 \text { r101 }}\right)$ & $0.0010 \pm 0.0022$ & $0.0027 \pm 0.0030$ & 0.0003 \\
$p_{n}\left(w_{c 101 \text { r101 }}\right)$ & $0.0015 \pm 0.0023$ & $0.0041 \pm 0.0039$ & 0.0061 \\
$p_{n}\left(w_{c 110 \text { r101 }}\right)$ & $0.0008 \pm 0.0014$ & $0.0025 \pm 0.0028$ & 0.0022 \\
$p_{n}\left(w_{c 001 \text { r101 }}\right)$ & $0.0008 \pm 0.0017$ & $0.0021 \pm 0.0024$ & 0.0107 \\
$p_{n}\left(w_{\text {c001 r110 }}\right)$ & $0.0115 \pm 0.0065$ & $0.0163 \pm 0.0065$ & 0.0201 \\
$p_{n}\left(w_{c 010 \text { r100 }}\right)$ & $0.0213 \pm 0.0131$ & $0.0301 \pm 0.0131$ & 0.0158 \\
$p_{n}\left(w_{\text {c011 r101 }}\right)$ & $0.0007 \pm 0.0011$ & $0.0024 \pm 0.0031$ & 0.0082 \\
$p_{n}\left(w_{c 100 \text { r010 }}\right)$ & $0.0007 \pm 0.0014$ & $0.0022 \pm 0.0028$ & 0.0012 \\
\hline
\end{tabular}

Sampling frequency $f_{\mathrm{s}}=1 \mathrm{~Hz}$ and $p_{\mathrm{g}}=$ global $p$ value.

TABLE 3. Probabilities of the words from the respiratory series and the global statistical significant level $p_{\mathrm{g}}$ between groups $S$ and $F$.

\begin{tabular}{lccc}
\hline & $\begin{array}{c}\text { Group S, } \\
\text { mean } \pm \text { SD }\end{array}$ & $\begin{array}{c}\text { Group F, } \\
\text { mean } \pm \text { SD }\end{array}$ & $p_{\text {g Value }}$ \\
\hline$p_{n}\left(w_{\text {rooo }}\right)$ & $0.240 \pm 0.054$ & $0.197 \pm 0.059$ & 0.0012 \\
$p_{n}\left(w_{\text {roo1 }}\right)$ & $0.109 \pm 0.023$ & $0.128 \pm 0.023$ & 0.0003 \\
$p_{n}\left(w_{\text {ro10 }}\right)$ & $0.005 \pm 0.010$ & $0.015 \pm 0.016$ & 0.0007 \\
$p_{n}\left(w_{\text {ro11 }}\right)$ & $0.112 \pm 0.024$ & $0.132 \pm 0.026$ & 0.0002 \\
$p_{n}\left(w_{\text {r10o }}\right)$ & $0.109 \pm 0.022$ & $0.128 \pm 0.023$ & 0.0003 \\
$p_{n}\left(w_{\text {r101 }}\right)$ & $0.007 \pm 0.003$ & $0.020 \pm 0.018$ & 0.0003 \\
$p_{n}\left(w_{\text {r110 }}\right)$ & $0.112 \pm 0.024$ & $0.133 \pm 0.026$ & 0.0002 \\
$p_{n}\left(w_{\text {r111 }}\right)$ & $0.303 \pm 0.068$ & $0.245 \pm 0.072$ & 0.0005 \\
\hline
\end{tabular}

Sampling frequency $f_{\mathrm{s}}=1 \mathrm{~Hz}$.

respiratory series for $f_{\mathrm{s}}=1 \mathrm{~Hz}$. The words from the cardiac series did not present significant statistical differences between both groups, independently of the sampling frequency. Table 3 shows the mean $\pm \mathrm{SD}$ of the word probabilities from the respiratory series for $f_{\mathrm{s}}=1 \mathrm{~Hz}$, and the statistical significant level $p_{\mathrm{g}}$ between groups $\mathrm{S}$ and $\mathrm{F}$. The most dominant words in both groups of patients were the same: patterns " 000 " and "111," with a higher probability of occurrence in group $\mathrm{S}$ than in group $\mathrm{F}$. The words containing "010" and "101" patterns presented a probability of occurrence nearly three times higher in group $\mathrm{F}$ than in group $\mathrm{S}$.

Table 4 shows the best results obtained when considering the number of words whose probability of occurrence was higher than a probability threshold and the number of forbidden words whose probability of occurrence was lower than a probability threshold, for $f_{\mathrm{s}}=1 \mathrm{~Hz}$. In group $\mathrm{S}$, there were more forbidden words than in group F. In group S, there were also
TABLE 4. Number of words whose probability of occurrence is higher than a probability threshold and number of forbidden words.

\begin{tabular}{lrrr}
\hline & $\begin{array}{c}\text { Group S, } \\
\text { mean } \pm \text { SD }\end{array}$ & \multicolumn{1}{c}{$\begin{array}{c}\text { Group F, } \\
\text { mean } \pm \text { SD }\end{array}$} & $p_{\mathrm{g}}$-value \\
\hline$f w_{c r} 1$ & $13.20 \pm 5.30$ & $7.92 \pm 6.66$ & 0.0001 \\
$f w_{c}, 2$ & $16.21 \pm 5.02$ & $11.59 \pm 6.63$ & 0.0004 \\
$f w_{c}, 3$ & $18.71 \pm 5.00$ & $14.51 \pm 6.30$ & 0.0003 \\
$f w_{r} 2$ & $1.84 \pm 0.47$ & $1.21 \pm 0.89$ & $<0.0001$ \\
$f w_{r} 3$ & $1.91 \pm 0.38$ & $1.51 \pm 0.79$ & 0.0002 \\
$w_{r} 20$ & $1.67 \pm 0.61$ & $1.15 \pm 0.87$ & 0.0025 \\
$w_{t} 25$ & $1.26 \pm 0.79$ & $0.56 \pm 0.75$ & $<0.0001$ \\
\hline
\end{tabular}

Sampling frequency $f_{\mathrm{s}}=1 \mathrm{~Hz}$ and $p_{\mathrm{g}}=$ global $p$ value.

more words than in group $\mathrm{F}$ whose probability of occurrence was higher than a probability threshold. It means that the histograms of the occurrence probability of the cardiorespiratory words and respiratory words presented a higher homogeneity in group $\mathrm{F}$ than in group $\mathrm{S}$.

One example of $R R(n)$ and $T_{T o t}(n)$ series of a patient is depicted in Fig. 6. Figures $7 \mathrm{a}$ and $7 \mathrm{~b}$ show an example of the surrogate series constructed in the case of univariate symbolic analysis of the series shown in Fig. 6, and Figs. $7 \mathrm{c}$ and $7 \mathrm{~d}$ in the case of bivariate joint symbolic analysis, following the methodology described previously. The obtained results, in both cases of univariate analysis and joint symbolic analysis, allow the rejection of the null hypothesis that a pattern is present only by chance.

In the last part of this study, we investigate if discriminant functions are able to discriminate multivariately between group $\mathrm{S}$ and group $\mathrm{F}$. Different discriminant functions of one or two variables were constructed using the most significant parameters from the time-domain analysis and the symbolic dynamic analysis. The leave-one-out procedure was used. Table 5 presents a summary of the results achieved using the variables from the time-domain analysis. The optimum percentage of correctly classified patients is $65.4 \%$.

The best results applying the symbolic dynamic analysis were mainly obtained for the sampling frequency of $1 \mathrm{~Hz}$. Table 6 presents a summary of the achieved discriminant analysis results. Accuracy is the percentage of correctly classified patients. The variables obtained with the symbolic dynamic analysis presented better discriminant results than the best variables proposed from the time-domain analysis. The optimum percentages of correctly classified patients were $78.2 \%$ and were obtained with the pair of variables $f w_{r} 3$ and $p_{n}\left(w_{c 110} r_{010}\right)$, as well as the pair of variables $p_{n}\left(w_{r 010}\right)$ and $p_{n}\left(w_{c 100} r_{101}\right)$. 

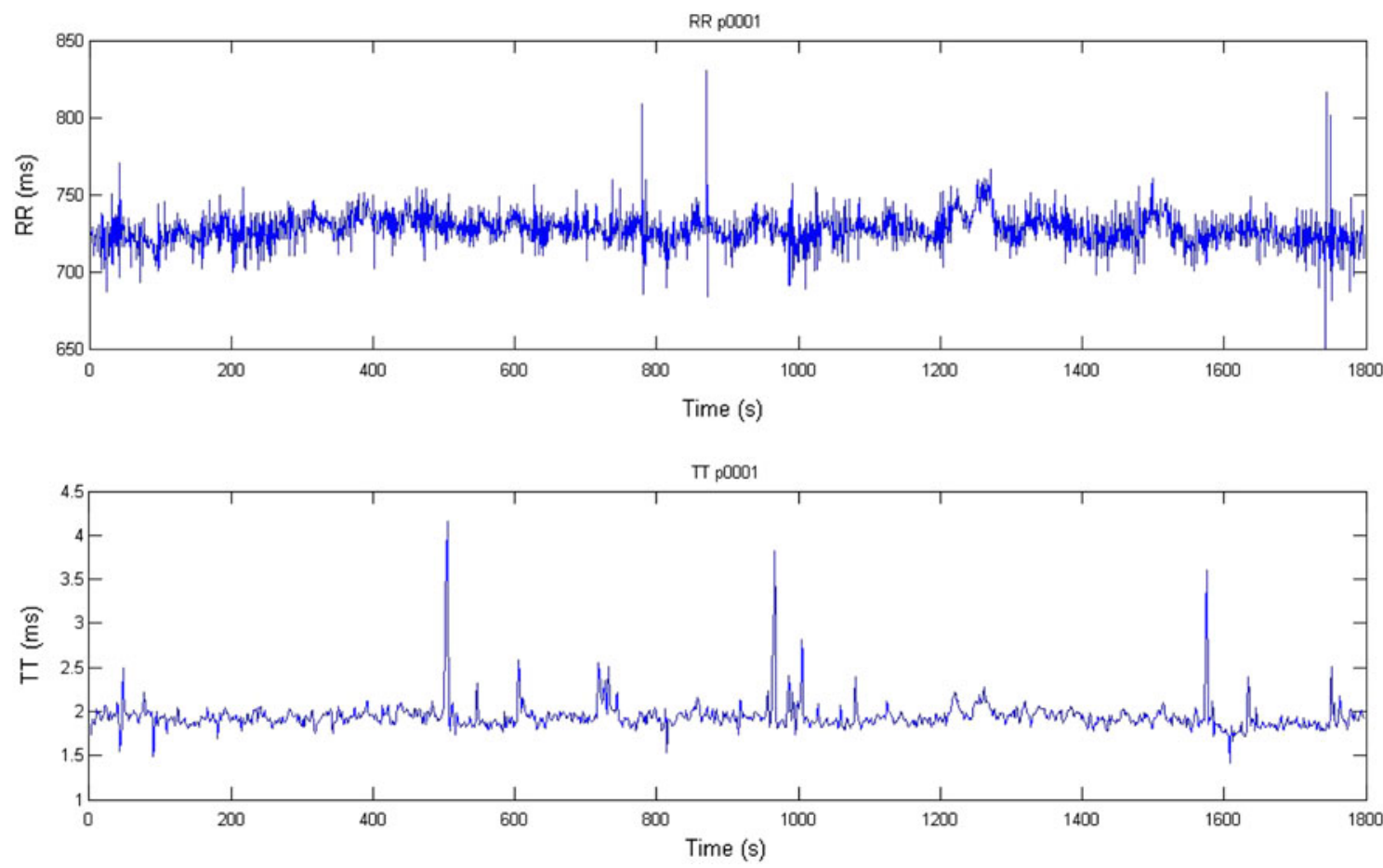

FIGURE 6. $R R(n)$ and $T_{T o t}(n)$ series of a patient.

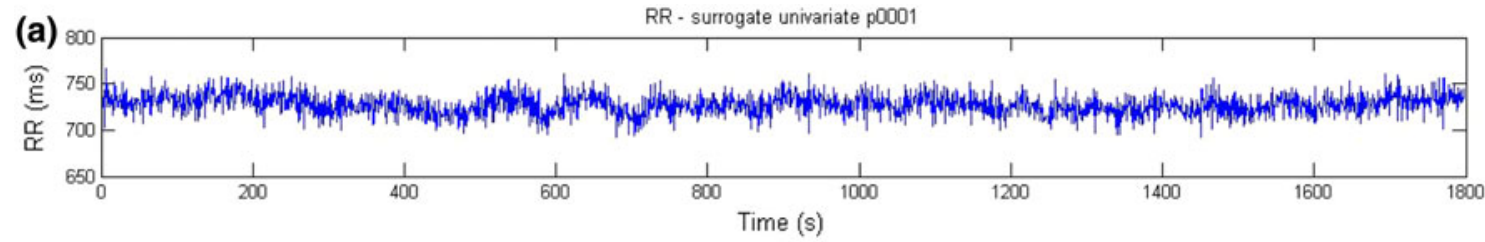

(b)
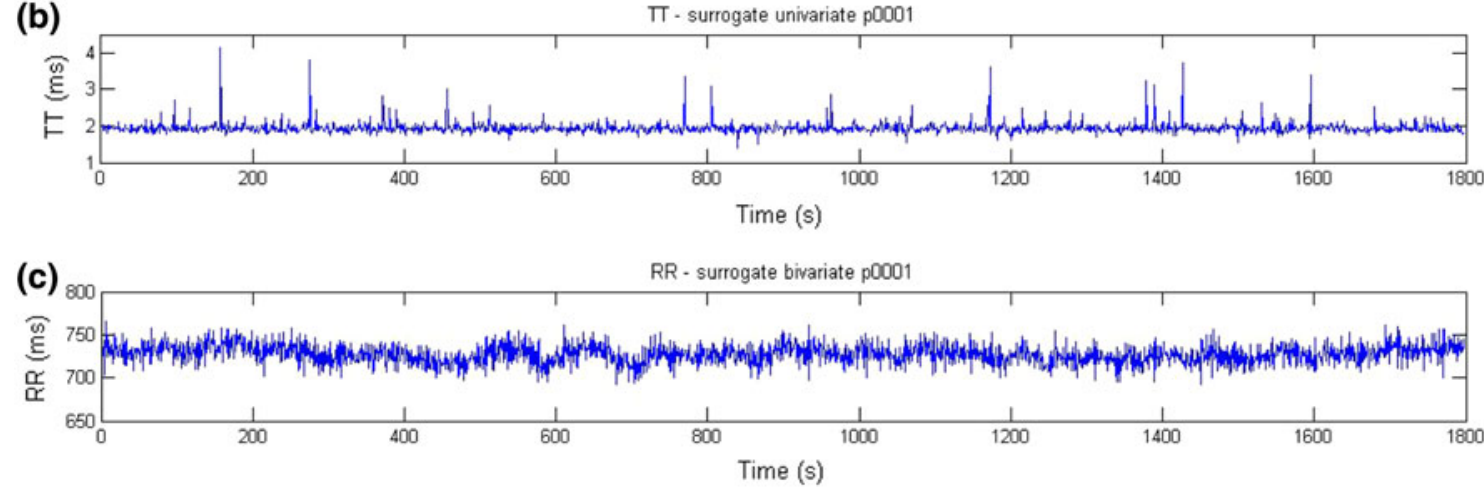

(d)

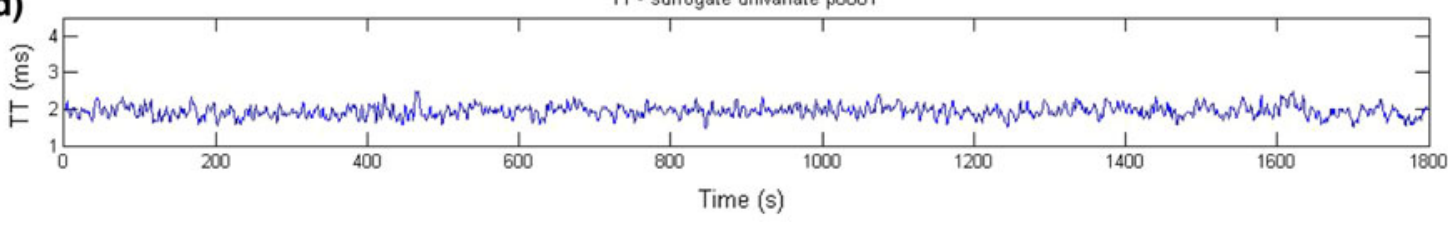

FIGURE 7. Surrogate series constructed in the cases of univariate (a and b) and bivariate (c and d) from the series shown in Fig. 6. 
TABLE 5. Discriminant analysis between the patient groups $S$ and $F$ using variables from the time-domain analysis.

\begin{tabular}{lc}
\hline & Accuracy $(\%)$ \\
\hline Mean $R R, S D\left(T_{\text {Tot }}\right)$ & 65.4 \\
Mean $T_{\text {Tot }}$ & 64.7 \\
Mean $T_{\text {Tot }}$ IQR $\left(T_{\text {Tot }}\right)$ & 64.7 \\
Mean RR, Mean $T_{T o t}$ & 63.9 \\
Mean $T_{\text {Tot }}, S D\left(T_{\text {Tot }}\right)$ & 63.9 \\
Mean RR & 62.4 \\
\hline
\end{tabular}

TABLE 6. Discriminant analysis between the patient groups $S$ and $F$ using variables from the symbolic dynamic analysis.

\begin{tabular}{lc}
\hline & Accuracy $(\%)$ \\
\hline$f w_{r} 3, p_{n}\left(w_{c 110}\right.$ r010 $)$ & 78.2 \\
$p_{n}\left(w_{r 010}\right), p_{n}\left(w_{c 100} r 101\right)$ & 78.2 \\
$p_{n}\left(w_{r 101}\right), p_{n}\left(w_{r 010}\right)$ & 76.7 \\
$f w_{r} 2$ & 76.7 \\
$p_{n}\left(w_{c 110}\right.$ r010 $)$ & 76.7 \\
$f w_{r} 3$ & 75.9 \\
$p_{n}\left(w_{c 100}\right.$ r101 $)$ & 75.9 \\
$p_{n}\left(w_{r 101}\right)$ & 75.9 \\
$p_{n}\left(w_{r 010}\right)$ & 75.2 \\
\hline
\end{tabular}

\section{DISCUSSION AND CONCLUSIONS}

The main objective of this paper was to improve the characterization of the complex dynamics of the cardiorespiratory interdependencies during the weaning procedure. Two groups of patients were considered: 94 patients with successful weaning and 39 patients that failed to maintain spontaneous breathing in the weaning procedure.

In order to show the advantage of using symbolic analysis, instead of more traditional and simpler linear tools, the relation between cardiac and breath cycles was previously analyzed using the cross-correlation coefficient $\rho_{c r}$. No statistical significant differences were obtained when comparing both groups of patients with this linear correlation technique.

The cardiac and the respiratory systems are controlled by two interacting self-sustained oscillators, each with its own period. The cardiorespiratory interactions of patients were studied by analyzing the nonlinear dynamics of the HR and the respiratory frequency. In this way, the cardiac interbeat durations and the breath durations, corresponding to the periods of both oscillators, were analyzed by symbolic dynamics. For this purpose, a linear interpolation method was performed, and a methodology was developed to characterize the different cardiorespiratory interactions involved in the weaning procedure. The use of higher order, or spline interpolation methods could possibly lead to different outcomes.
The influences of the parameters that were used in the proposed symbolic dynamic analysis were analyzed: the number of overlapped symbols in consecutive words and the sampling frequency. The most suitable parameter values were selected for the study of the cardiorespiratory interactions.

The word distribution matrix $W$ enables a coarsegrained quantitative assessment of short-term nonlinear analysis of the cardiorespiratory interdependencies. The word distribution within $W$ clearly showed the presence of deterministic structures in both groups of patients, because stochastically independent HR and respiratory dynamic patterns would result in a more or less equal distribution. The four most dominant words presented monotonous patterns in the BR (000 or 111) and alternant patterns in the cardiac rate (010 or 101). A larger presence of words 000 and 111 from the respiratory series in patients who were successful in weaning procedure reflects a lower variability of the BRs. The presence of alternant patterns in heart period variability has been linked to vagal modulation. ${ }^{27}$ Since in group F the presence of alternant patterns from the cardiac series decreased with respect to group $\mathrm{S}$, it can be concluded that vagal withdrawal is a sign of discomfort.

When analyzing the probabilities of the words from the respiratory series (Table 3 ), the most dominant words were the ones with monotonous patterns 000 and 111, in both groups of patients, with a probability of occurrence higher in the group $\mathrm{S}$ than within the group $\mathrm{F}$, reflecting a lower variability of the BRs in group S. The words containing alternant patterns $(010$ and 101) presented a probability of occurrence nearly three times greater in group $\mathrm{F}$ compared to group $\mathrm{S}$, reflecting a higher variability of the BRs in group F. Significant differences between both groups occurred in all the word types from the respiratory series, while the words from the cardiac series did not present significant statistical differences. Respiratory center responds mainly with tachyapnea in front of acute respiratory failure. This fact may justify that the time intervals of respiratory pattern obtained more significant results than the time intervals of cardiac pattern.

The histograms of the occurrence probability of the cardiorespiratory words presented a lower homogeneity in the group of patients with successful trials when compared with the group of patients that failed to maintain spontaneous breathing. This was confirmed by a higher number of words whose probability of occurrence was higher than a probability threshold in group $\mathrm{S}$ as well as a higher number of forbidden words in group S (Table 4). It means the presence of stronger deterministic structures in patients who were successful in weaning procedures, and reflects a stronger coupling between cardiac and respiratory oscillators in these patients. It can be suggested that this stronger 
interaction between heart period and respiration results in an increase of respiratory sinus arrhythmia. In addition, a lower homogeneity reflects a stronger coupling between the cardiac and respiratory oscillators because stochastically independent series would result in a more o less equal distribution.

The occurrence probability of the cardiorespiratory word types that presented a global statistical significance was nearly three times greater in group $F$ than in group S. Discriminant functions of one or two variables were constructed using the most significant parameters from the time-domain analysis and the symbolic dynamic analysis. In this discriminant analysis, the variables from the symbolic dynamic analysis revealed better results than the best variables from the time-domain analysis. The optimum discriminant functions of one variable were constructed using parameters obtained from the symbolic dynamic analysis of the respiratory series. It means that when considering only one kind of time series the respiratory patterns are more determinant. However, the discriminant functions of two variables, obtained from the cardiorespiratory interaction analysis, allow a better classification of patients. The effect of changes in respiratory patterns, the effect of changes in autonomic control, or other, could be possible explanations on what the measured coupling really reflects in terms of underlying physiological mechanisms.

In conclusion, joint symbolic dynamics provides a new representation of $\mathrm{HR}$ and $\mathrm{BR}$ short-term interactions. For the first time, the cardiorespiratory interactions during the weaning procedure have been analyzed. Joint symbolic dynamics shows significant differences when comparing the patients with successful weaning and the patients that failed to maintain spontaneous breathing in the weaning procedure during the 30-min T-tube test. Therefore, this method could be suitable for reducing the number of patients that had to be reintubated before $48 \mathrm{~h}$ but previously performed successful trials during the 30-min test. These results should be validated with a group of reintubated patients that was not available during this study.

\section{ACKNOWLEDGMENTS}

This work was supported in part by Ministerio de Ciencia e Innovación under grants TEC2007-63637 and TEC2007-68076-C02-01 from the Spanish Government. The authors thank to Dr. A. Ballestero and his collaborators of the Hospital Universitario de Getafe, Spain, for their collaboration in the signal database acquisition.

\section{OPEN ACCESS}

This article is distributed under the terms of the Creative Commons Attribution Noncommercial License which permits any noncommercial use, distribution, and reproduction in any medium, provided the original author(s) and source are credited.

\section{REFERENCES}

${ }^{1}$ Angelini, L., R. Maestri, D. Marinazzo, L. Nitti, M. Pellicoro, G. D. Pinna, S. Stramaglia, and S. A. Tupputi. Multiscale analysis of short term heart beat interval, arterial blood pressure and instantaneous lung volume time series. Artif. Int. Med. 41:237-250, 2007.

${ }^{2}$ Baumeister, B., M. F. El-Khatib, P. G. Smith, and J. L. Blumer. Evaluation of predictors of weaning from mechanical ventilation in pediatric patients. Pediatr. Pulmonol. 24:344-352, 1997.

${ }^{3}$ Baumert, M., T. Walther, J. Hopfe, H. Stepan, R. Faber, and A. Voss. Joint symbolic dynamic analysis of beat-tobeat interactions of heart rate and systolic blood pressure in normal pregnancy. Med. Biol. Eng. Comput. 40:241-245, 2002.

${ }^{4}$ Boles, J. M., J. Bion, A. Connors, M. Herridge, B. Marsh, C. Melote, R. Pearl, H. Silverman, M. Stanchina, A. Vieillard-Baron, and T. Welte. TASK FORCE-weaning from mechanical ventilation. Eur. Respir. J. 29:1033-1056, 2007.

${ }^{5}$ Caminal, P., B. Giraldo, H. Zabaleta, M. Vallverdú, S. Benito, D. Ballesteros, L. López-Rodriguez, A. Esteban, M. Baumert, and A. Voss. Joint symbolic dynamic analysis of cardiorespiratory interactions in patients on weaning trial. In: Proc. 27th International Conference of the IEEE Engineering in Medicine and Biology Society, 2005, CD ROM, p. 1239

${ }^{6}$ Caminal, P., M. Vallverdú, B. Giraldo, S. Benito, G. Vázquez, and A. Voss. Optimized symbolic dynamics approach for the analysis of the respiratory pattern. IEEE Trans. Biomed. Eng. 52:1832-1839, 2005.

${ }^{7}$ Caroleo, S., F. Agnello, K. Abdallah, E. Santangelo, and B. Amantea. Weaning from mechanical ventilation: an open issue. Minerva Anestesiol. 73:417-427, 2007.

${ }^{8}$ Casaseca, P., M. Martín, and C. Alberola. Weaning from mechanical ventilation: a retrospective analysis leading to a multimodal perspective. IEEE Trans. Biomed. Eng. 53:1330-1345, 2006.

${ }^{9}$ Censi, F., G. Calcagnini, S. Lino, S. R. Seydnejad, R. I. Kitney, and S. Cerutti. Transient phase locking patterns among respiration, heart rate and blood pressure during cardiorespiratory synchronisation in humans. Med. Biol. Eng. Comput. 38:416-426, 2000.

${ }^{10}$ del Rosario, N., C. S. Sassoon, K. G. Chetty, S. E. Gruer, and C. K. Mahutte. Breathing pattern during acute respiratory failure and recovery. Eur. Respir. J. 10(11):25602565, 1997.

${ }^{11}$ El-Khatib, M. F. A diagnostic software tool for determination of complexity in respiratory pattern parameters. Comput. Biol. Med. 37:1522-1727, 2007.

${ }^{12}$ Faes, L., A. Porta, R. Cucino, S. Cerutti, R. Antolini, and G. Nollo. Causal transfer function analysis to describe closed loop interactions between cardiovascular and 
cardiorespiratory variability signals. Biol. Cybernet. 90: 390-399, 2004.

${ }^{13}$ Hendra, K. P., and B. R. Celli. Weaning from mechanical ventilation. Int. Anest. Clin. 37:127-143, 1999.

${ }^{14}$ Hlavackova-Schindler, K., M. Palus, and M. Vejmelka. Causality detection based on information-theoretic approaches in time series analysis. Phys. Rep. Rev. Sect. Phys. Lett. 441:1-46, 2007.

${ }^{15}$ Hoyer, D., R. Bauer, B. Walter, and U. Zwiener. Estimation of nonlinear couplings on the basis of complexity and predictability - a new method applied to cardiorespiratory coordination. IEEE Trans. Biomed. Eng. 45:545-552, 1998.

${ }^{16}$ Hoyer, D., U. Leder, H. Hoyer, B. Pompe, M. Sommer, and U. Zwiener. Mutual information and phase dependencies: measures of reduced nonlinear cardiorespiratory interaction after myocardial infarction. Med. Eng. Phys. 24:33-43, 2002.

${ }^{17}$ Kurths, J., A. Voss, P. Saparin, A. Witt, H. J. Kleiner, and N. Wessel. Quantitative analysis of heart rate variability. Med. Eng. Phys. 5(1):88-04, 1995.

${ }^{18}$ MacIntyre, N. R. Evidence-based guidelines for weaning and discontinuing ventilatory support. Chest 120:375-395, 2001.

${ }^{19}$ Martinez, J. P., R. Almeida, S. Olmos, A. P. Rocha, and P. Laguna. A wavelet-based ECG delineator: evaluation on standard databases. IEEE Trans. Biomed. Eng. 51:570-581, 2004.

${ }^{20}$ Musizza, B., A. Stefanovska, M. Palus, J. Petrovic, and F. Bajrovic. Interactions between cardiac, respiratory and EEG oscillations in rats during anaesthesia. J. Physiol. 580:315-326, 2007.

${ }^{21}$ Novak, V., P. Novak, J. de Champlain, A. R. Le Blanc, R. Martin, and R. Nadeau. Influence of respiration on heart rate and blood pressure fluctuations. J. Appl. Physiol. 74(2):617-626, 1993

${ }^{22}$ Penzel, T., N. Wessel, M. Riedl, J. W. Kantelhardt, and S. Rostig. Cardiovascular and respiratory dynamics during normal and pathological sleep. Chaos 17:15116, 2007.

${ }^{23}$ Pompe, B., P. Blidh, D. Hoyer, and M. Eiselt. Using mutual information to measure coupling in the cardiorespiratory system. IEEE Eng. Med. Biol. Mag. 17:32-39, 1998.

${ }^{24}$ Porta, A., G. Baselli, S. Guzzetti, M. Pagani, A. Malliani, and S. Cerutti. Prediction of short cardiovascular variability signals based on conditional distribution. IEEE Trans. Biomed. Eng. 47:1555-1564, 2000.

${ }^{25}$ Porta, A., S. Guzzetti, N. Montano, R. Furlan, M. Pagani, A. Malliani, and S. Cerutti. Entropy, entropy rate, and pattern classification as tools to typify complexity in short heart period variability series. IEEE Trans. Biomed. Eng. 48:1282-1291, 2001.

${ }^{26}$ Porta, A., S. Guzzetti, N. Montano, M. Pagani, V. Somers, A. Malliani, G. Baselli, and S. Cerutti. Information domain analysis of cardiovascular variability signals: evaluation of regularity, synchronization and co-ordination. Med. Biol. Eng. Comput. 38:180-188, 2000.

${ }^{27}$ Porta, A., E. Tobaldini, S. Guzzetti, R. Furlan, N. Montano, and T. Gnecchi-Ruscone. Assessment of cardiac autonomic modulation during graded head-up tilt by symbolic analysis of heart rate variability. Am. J. Physiol. Heart Circ. Physiol. 293(1):H702-H708, 2007.

${ }^{28}$ Randolph, A. G., D. Wypij, S. T. Venkataraman, J. H. Hanson, and J. H. Arnold. Effect of mechanical ventilator weaning protocols on respiratory outcomes in infants and children. JAMA 288(20):2561-2568, 2002.

${ }^{29}$ Schafer, C., M. G. Rosemblum, J. Kurths, and H. H. Abel. Heartbeat synchronized with ventilation. Nature 392:239240, 1998

${ }^{30}$ Sellares, J., I. Acerbi, H. Loureiro, R. L. Dellaca, M. Ferrer, A. Torres, D. Navajas, and R. Farre. Respiratory impedance during weaning from mechanical ventilation in a mixed population of critically ill patients. $\mathrm{Br} . J$. Anaesth. 103:828-832, 2009.

${ }^{31}$ Task Force of the Europe Society of Cardiology and the North American Society of Pacing and Electrophysiology. Heart rate variability: standards of measurement, physiological interpretation and clinical use. Circulation 93(5): 1043-1065, 2009.

${ }^{32}$ Theiler, J., S. Eubank, A. Longtin, and J. Galdrikian. Testing for nonlinearity in time series: the method of surrogate data. Physica D 58:77-94, 1992.

${ }^{33}$ Tinsley, H. E. A., and Steven D. Brown, editors. Handbook of Applied Multivariate Statistics and Mathematical Modeling. London: Academic Press, 2000.

${ }^{34}$ Tobin, M. J. Advances in mechanical ventilation. N. Engl. J. Med. 344:1986-1996, 2001.

${ }^{35}$ Toledo, E., S. Akselrod, I. Pinhas, and D. Aravot. Does synchronization reflect a true interaction in the cardiorespiratory system? Med. Eng. Phys. 24:45-52, 2002.

${ }^{36}$ Vassilakopoulos, T., C. Roussos, and Z. Spyros. Weaning from mechanical ventilation. J. Crit. Care 14:39-62, 1999.

${ }^{37}$ Voss, A., J. Kurths, H. J. Kleiner, A. Witt, N. Wessel, P. Saparin, K. J. Osterziel, R. Schurath, and R. Dietz. The application of methods of non-linear dynamics for the improved and predictive recognition of patients threatened by sudden cardiac death. Cardiovasc. Res. 31:419-433, 1996.

${ }^{38}$ Voss, A., S. Schulz, R. Schroeder, M. Baumert, and P. Caminal. Methods derived from nonlinear dynamics for analysing heart rate variability. Philos. Transact. A Math. Phys. Eng. Sci. 367:277-296, 2008.

${ }^{39}$ Yang, K. L., and M. J. Tobin. A prospective study of indexes predicting the outcome of trials of weaning from mechanical ventilation. N. Engl. J. Med. 324:1445-1450, 1991. 